\title{
BIOACTIVE GLASS IN CAVITARY BONE DEFECTS: A COMPARATIVE EXPERIMENTAL STUDY IN RABBITS
}

\author{
André Ferrari de França $C_{a m a r g o}{ }^{1}$, André Mathias Baptista ${ }^{1}$, Renato Natalino ${ }^{1}$, Olavo Pires de Camargo ${ }^{2}$
}

\section{ABSTRACT}

Objectives: To compare bioactive glass and autograft regarding their histomorphometric characteristics. Methods: The authors conducted a prospective case-control experimental study on animals in order to compare the histomorphometric characteristics of bioactive glass versus autograft. Eight rabbits underwent surgery in which a cavitary defect was created in both proximal femurs. One side was filled with bioactive glass granules and the other, with autograft grafted from the contralateral side. The sides were randomized. Fourteen days after surgery, the animals were eutha- nized. Results: Histologic analysis revealed that bone neoformation was equivalent among the two groups and the osteoblasts cell-count was higher in the femurs treated with bioactive glass. The osteocytes cell-count, however, was lower. The similarity in bone formation between both groups was consistent to literature findings. Conclusion: Bioactive glass is similar to autograft regarding bone neoformation in this animal model of cavitary bone defects. Level of Evidence III, Case-Control Study.

Keywords: Bone transplantation. Osteogenesis. Rabbits.

Citation: Camargo AFF, Baptista AM, Natalino R, Camargo OP. Bioactive glass in cavitary bone defects: a comparative experimental study in rabbits. Acta Ortop Bras. [online]. 2015;23(4):202-7. Available from URL: http://www.scielo.br/aob.

\section{INTRODUCTION}

The reconstruction of the bone defects remains a technical challenge for modern medicine. ${ }^{1}$ Bone defects can occur due to various situations, as traumatic bone losses, osteomyelitis and bone tumors.

Bone autograft, due to its osteoconduction, osteoinduction, and osteogenesis properties is still considered the golden standard. ${ }^{1,2}$ Meanwhile, its use presents some limitations: there is limited availability (since it is an exhausting source) and causes morbidity in the donor site, as scars and postoperative pain, besides the risk of surgical complications in the donor site, that can occur in up to $10 \%$ of cases. ${ }^{3,4}$ Moreover, the biological quality of autograft depends on the patient's overall health status and age. ${ }^{5}$

These difficulties and limitations have motivated the search for bone substitutes that would be an alternative for autologous graft. Bioactive glass is one of the subgroups of synthetic biomaterials made of silica $\left(\mathrm{SiO}_{2}\right)$. Its basic molecular structure is a tetrahedron formed by a silicon atom in the center with four equidistant oxygen atoms in the vertices. (Figure 1)

Bioactive glasses have unique binding properties to the bone surface $^{6,7}$ and its main characteristic is the capacity to promote a chemical bond with hydroxyapatite and generate a framework for bone growth. ${ }^{8}$
Bioactive glass used in this study is the $553 \mathrm{P} 4\left(53 \% \mathrm{SiO}_{2}, 23 \%\right.$ $\mathrm{Na}_{2} \mathrm{O}, 20 \% \mathrm{CaO}$, and $4 \% \mathrm{P}_{2} \mathrm{O}_{5}$ ) which brand name is BonAlive ${ }^{\circledR}$. It is a synthetic bone substitute with osteoinduction, $, 9,10$ and osteoconduction ${ }^{11}$ properties, which is also anti-bacterial, ${ }^{10,12,13}$ a property which probably results from high $\mathrm{pH}$ and osmotic effect induced by the glass solubilization. ${ }^{10}$

The bioactive glass granules stimulate the growth and maturation of osteoblasts, besides promoting the expression and maintenance of the osteoblast phenotype (by delaying differentiation into osteocyte). The bioactive glass also stimulates the increase of osteoblasts function, making them more active. ${ }^{7}$ The hypothesis of this study is that bone neoformation promoted by bioactive glass in repairing cavitary bone injuries is equivalent to bone neoformation provided by autograft. As the bioactive glass induces an increase of the rate of local bone turnover, ${ }^{7}$ we expect to find an increase in the number of osteoblasts and osteoclasts in the cases exposed to the bioactive glass. Moreover, we expect a drop in the osteocyte count, since the bioactive glass slow down the differentiation of osteoblasts into osteocytes. ${ }^{7}$ There is little information on the literature regarding the use of the bioactive glass S53P4 in cavitary injuries, using histopathological analysis to compare it to autograft, still considered a graft standard. ${ }^{1}$ Therefore, the knowledge about cellular and molecular biology mechanisms involved in this process are yet limited.

All the authors declare that there is no potential conflict of interest referring to this article.

\footnotetext{
1. Universidade de São Paulo, Hospital das Clínicas da Faculdade de Medicina, Instituto de Ortopedia e Traumatologia do, São Paulo, SP, Brazil. 2. Universidade de São Paulo, Faculdade de Medicina, Department of Orthopedics and Traumatology, São Paulo, SP, Brazil.

Work developed at Universidade de São Paulo, Faculdade de Medicina, Laboratory of Medical Investigation of the Musculoskeletal System, Department of Orthopedic and Traumatology, São Paulo, SP, Brazil.

Correspondence: Rua Dr. Ovídio Pires de Campos, 333, $3^{\circ}$ andar, São Paulo, SP, Brazil. andre.ferrari@hc.fm.usp.br
} 


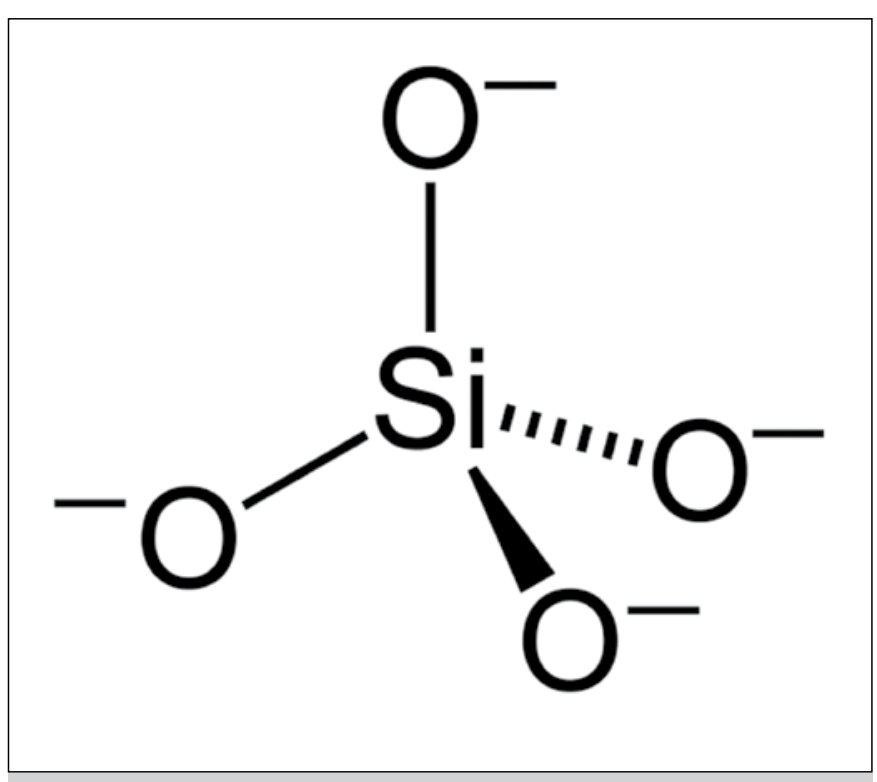

Figure 1. Basic molecular structure of $\mathrm{SiO}_{4}$ - the basis for all kinds of silicates. The vertices of the tetrahedron are four oxygen atoms; the silicon atom is located at the exact center.

The objective of this work was to compare histological characteristics of bone neoformation provided by bioactive glass in the repair of cavitary bone injuries and autograft, considered the gold standard.

\section{METHODS}

The bioactive glass selected for this study was S53P4, based on Lindfords' study. ${ }^{14}$ The granules are produced under the brand name BonAlive ${ }^{\circledR}$ by the Center of Biological Materials in Turku, Finland, with size between $630-800 \mu \mathrm{m}$. The material is formed by $53 \% \mathrm{SiO}_{2}, 23 \% \mathrm{Na}_{2} \mathrm{O}, 20 \% \mathrm{CaO}$ and $4 \% \mathrm{P}_{2} \mathrm{O}_{5}$.

Male rabbits of the New Zealand lineage from the Central Animal Facility from Faculdade de Medicina da Universidade de São Paulo, SP, Brazil, were used in this study. The animals were raised in accordance to ethical standards and released by the central animal facility for the experiment. The animals were maintained in individual cages in controlled illumination, temperature, and moisture conditions, receiving water and specific animal food ad libitum. Before each operative procedure, they were submitted to eight hours fasting.

Through the preliminary results of the first three animals, calculation of the size of the sample was carried out using osteoblasts count values as quantitative parameter. With $80 \%$ power, the calculated sample size was eight rabbits.

The following exclusion criteria were adopted:

- Weight $<5 \mathrm{~kg}$ (according to observation in the pilot project, the use of smaller rabbits hindered the production of bone defects);

- Locomotion alterations or clinical issues;

- Infection, fracture in the site to be operated, or significant clinical changes, such as weight loss higher than $10 \%$ after the surgical procedure.

\section{Surgical technique}

Before anesthetizing the animals, the side that would receive the bioactive glass or the autograft was decided by sortition.
The animals were then anesthetized by the standard procedure and positioned in lateral decubitus, with the side that would receive the bioactive glass facing upwards. The surgical access was longitudinal, centered on the greater trochanter, with an extension enough to expose the trochanter (nearly $3 \mathrm{~cm}$ ) by dissection of the skin, subcutaneous, gluteus maximum and finally bone exposure. (Figure 2)

Using a delicate punch cutter tweezer, the side of the lateral cortex of the femur at the base of the greater trochanter was removed. Through this bone window, a cavity defect was created using a curette. The dimensions of the injury were $1 \mathrm{~cm}$ longitudinal extension (size of the curette), and transversally, the inner diameter of the femur (approximately $6.5 \mathrm{~mm}$ ); therefore, the defect was delimitated by the proximal femur cortex. (Figure 3) The removed bone was carefully collected and stored for use as autograft on the contralateral side. The created bone defect was filled with bioactive glass beads; (Figure 4) followed by closure of the muscle tissue with nylon stitches and suture of the skin. The animal had, then, its position altered and the same procedure was repeated on the contralateral side. However, the cavity defect this time was filled with the autograft that had been collected from the other side. After surgery, the rabbits were returned to their cages and kept in an environment with controlled temperature, humidity and circadian cycle, receiving food and water ad libitum. Total load bearing on the operated limbs was allowed immediately, without any kind of restriction or restraint. Two weeks after surgery, the animals were sacrificed by administration of potassium chloride solution. Samples were removed and sent for histopathological analysis.

The histomorphometric methodology employed was adapted from Rosselli's. ${ }^{15}$ Samples were fixed in buffered $10 \%$ formalin and subjected to decalcification with EDTA. The specimens were then plated in $5 \%$ aqueous sodium bicarbonate solution and were dehydrated with increasing ethanol concentrations diaphonized with xylene. Then, the specimens were cut in the direction of the coronal axis of the femur, in the central region of the bone defect, and then embedded in paraffin. Slides were

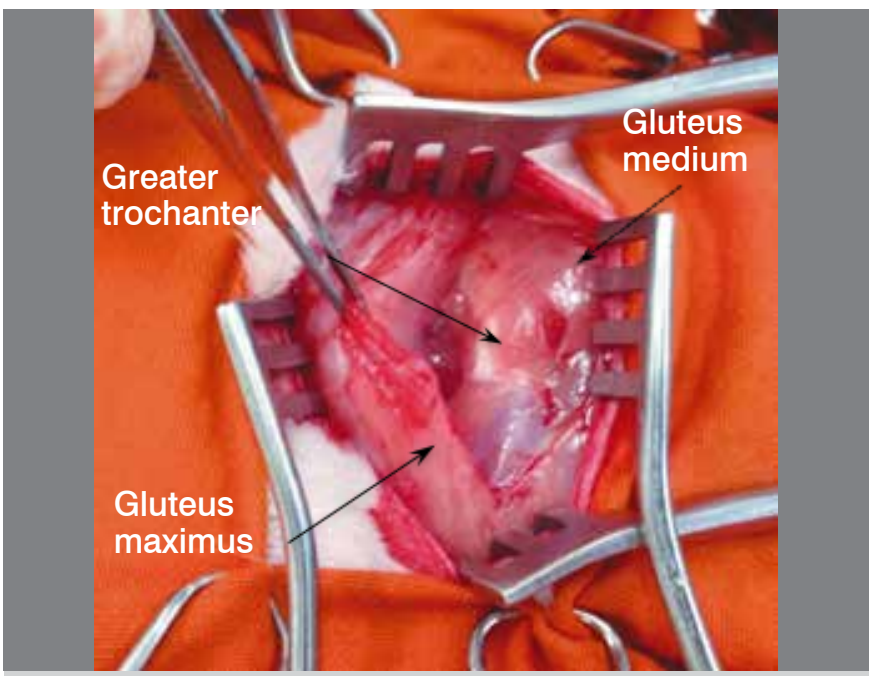

Figure 2. Surgical way of access; gluteus maximus set aside (tweezers) and exposure of the greater trochanter. The gluteus medium muscle tendons and internal rotators are kept untouched (rabbit 2 , right side). 


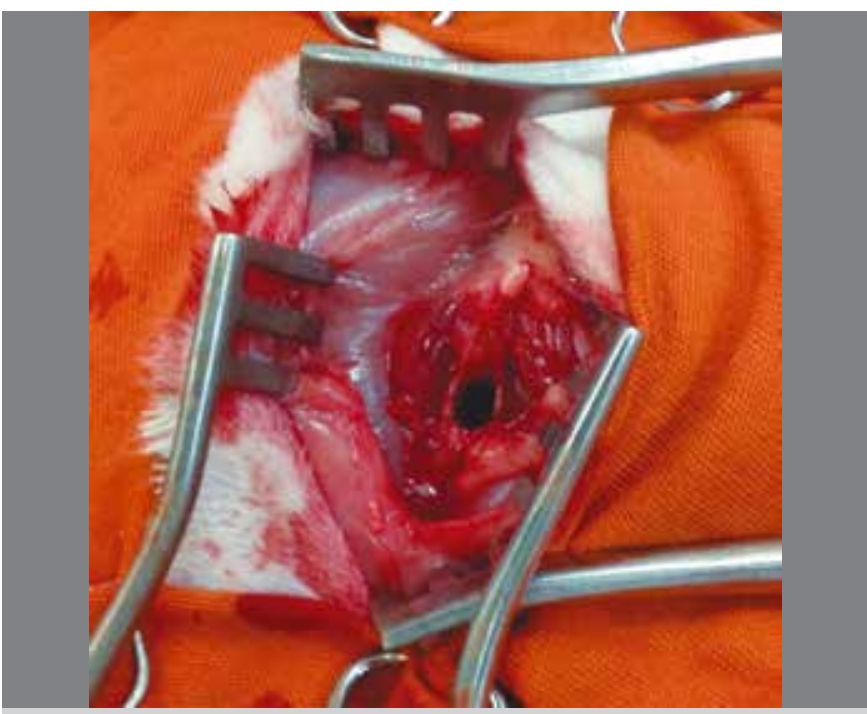

Figure 3. Bone defect already created, limited by the femur cortex (rabbit 2, right side).

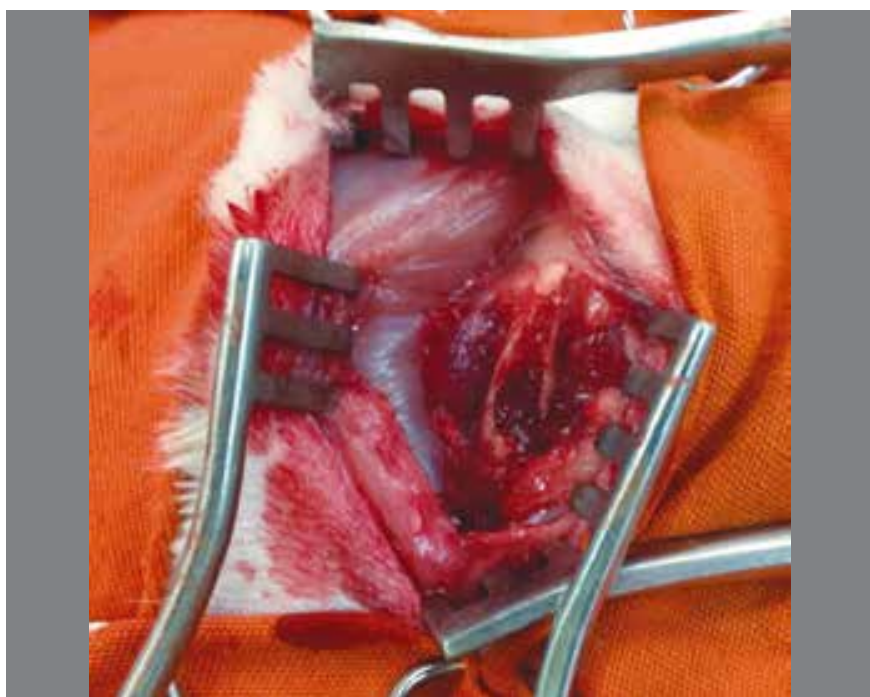

Figure 4. Bone defect filled with bioactive glass beads (rabbit 2, right side).

prepared with $5 \mu \mathrm{m}$ histological cuts and stained with hematoxylin-eosin (HE). The descriptive histological analysis of the slides was performed by light microscopy.

The following variables were analyzed: semi-quantitative variables (neovascularization, fibrosis, bone neoformation and inflammatory infiltrate); quantitative variables (longitudinal and transverse size of the bone defect creates, number of osteoblasts, osteocytes and osteoclasts). The evaluation of these parameters was performed by two observers on an Olympus CX41 microscope coupled to a digital Leica DC300F camera. Histological analysis was performed according to the presence and intensity of the aforementioned variables.

Each variable was considered as follows: neovascularization is the presence of neoformed capillaries and medium-caliber vessels in the bone defect region; fibrosis is the presence of dense connective tissue with collagen fibers; inflammatory infiltrate is the presence of inflammatory cells (lymphocytes and monocytes); bone neoformation is immature bone tissue with disorganized collagen arrangement. Osteoblasts are active (of polygonal appearance) or inactive (of flattened appearance) cells on the surface of the trabeculae; osteocytes are inactive cells incorporated into the bone matrix; osteoclasts are multinucleated giant cells found in gaps in the surface of the trabeculae (responsible for bone resorption).

Semi-quantitative variables were categorized in ascending scale between zero and three, as established by the Anatomic-Pathology Service of Instituto de Ortopedia e Traumatologia do Hospital das Clínicas da FMUSP, São Paulo, SP, Brazil, ${ }^{15}$ taking into account the intensity of the microscopic phenomena observed. (Table 1) Each slide was divided into four quadrants representing approximately $25 \%$ of the total area observed in the smallest increase. The average of the four quadrants scores represents the score given to the slide.

The presence and intensity of bone formation was categorized as: 0 , no neoformation; 1 , neoformation between 1 and $25 \%$ of the defect; 2 , neoformation between 26 and $50 \%$ of the defect; and 3, neoformation between 51 and $100 \%$. The presence and quantification of fibrosis, inflammatory infiltrate and new bone formation were categorized in the same way. The number of osteoblasts, osteoclasts, and osteocytes was counted in the same microscope using the greater increase objective (Olympus - Plan CN 40x - area of $0.17 \mathrm{~mm}^{2}$ ) in the area with the highest density of bone neoformation chosen by the pathologist.

\section{Statistical analysis}

The semi-quantitative assessment were described with absolute and relative frequencies in each femur and comparative among the femurs with the paired Wilcoxon test. ${ }^{16}$ The quantitative magnitudes were described with summary measures (mean, standard deviation, median, minimum and maximum) in each femur and compared by means of paired Student t-test, ${ }^{16}$ except the osteoclasts, that were also compared through the Wilcoxon paired test. The tests were performed with a level of significance of $5 \%$.

\section{RESULTS}

The results are summarized in Tables 2, 3 and 4. The average area of the defects created (longitudinal measure, in the center of the injury) was of $63.5 \mathrm{~mm}^{2}$ in the control group and $70.5 \mathrm{~mm}^{2}$ in the bioactive glass group, a difference that was not statistically significant. No difference was observed between the two groups regarding bone neoformation, neovascularization, and fibrosis. However, there was a tendency, although not significant, that

\begin{tabular}{c|c}
$\begin{array}{c}\text { Table 1. Score grades attributed to variables vascularization, fibrosis, } \\
\text { inflammatory infiltrate and reactional bone neoformation. }\end{array}$ \\
\hline Score & Area of the quadrant \\
\hline 0 - None & $0 \%$ \\
\hline 1 - Discrete & $<25 \%$ \\
\hline $2-$ Moderate & $26-50 \%$ \\
\hline $3-$ Intense & $>50 \%$ \\
\hline
\end{tabular}


femurs treated with bioactive glass have lower degrees of inflammatory infiltrate than those treated with autograft.

Regarding cell counts, we observed that femurs treated with bioactive glass, presented osteoblast counts greater than femurs treated with autograft, but the number of osteocytes was smaller. The number of osteoclasts was similar between the two groups. Figures 5-10 show histological microphotographs of femurs treated with either autograft or bioactive glass.

\section{DISCUSSION}

The autologous bone graft, or autograft, can be used in all cases presenting bone tissue loss, such as benign bone cavitary injuries. Nowadays it is still considered the gold standard procedure. However, there are some limitations for its use. The search by bone substitutes with qualities similar to the autograft's, but without its disadvantages, culminated in recent decades with the development of a new class of materials, the bioactive glasses. The main objective of this study was to compare the intensity of bone neoformation between the two groups, one treated with autograft and the other with bioactive glass granules. The equality

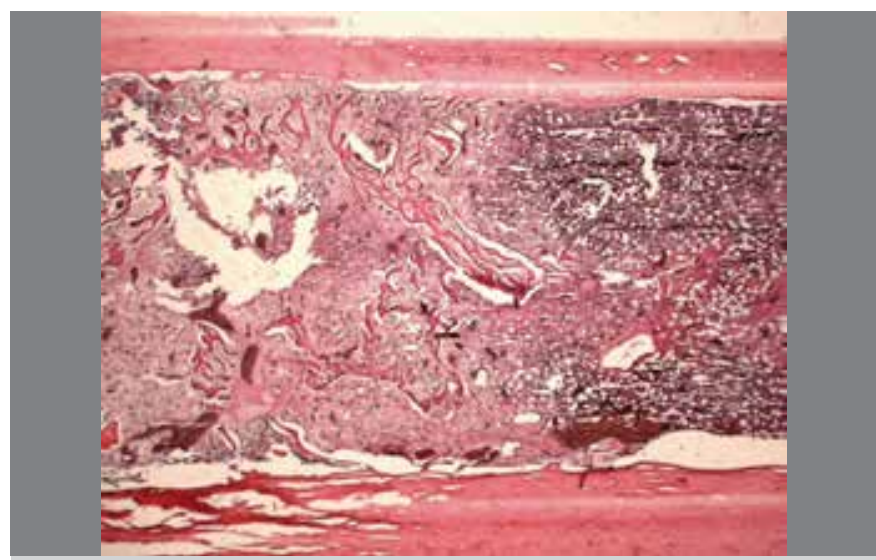

Figure 5. Example of a femur treated with autograft showing both cortex bones (above and below the picture), part of the created defect (left) and the normal bone (right). It is clearly shown the distinction between the two areas; to the right, there are few functioning trabeculae and the marrow (hematopoietic tissue) is exuberant; at left, there is plenty of fibrosis and new bone formation, with immature trabeculae. HE staining, 12.5x magnification (rabbit 2, left side).

Table 2. Individual data.

\begin{tabular}{|c|c|c|c|c|c|c|c|c|c|c|c|c|c|c|c|c|c|c|}
\hline \multicolumn{10}{|c|}{ Autograft } & \multicolumn{9}{|c|}{ Bioactive glass } \\
\hline 1 & $R$ & 57.8 & 66 & 60 & 4 & 3 & 2 & 0 & 1 & $L$ & 60.0 & 105 & 38 & 1 & 3 & 1 & 2 & 2 \\
\hline 2 & $L$ & 77.0 & 72 & 63 & 1 & 2 & 1 & 1 & 2 & $\mathrm{R}$ & 68.3 & 70 & 12 & 0 & 2 & 1 & 1 & 2 \\
\hline 3 & $\mathrm{R}$ & 69.0 & 53 & 47 & 1 & 2 & 2 & 2 & 2 & $L$ & 68.3 & 111 & 55 & 1 & 3 & 0 & 2 & 1 \\
\hline 6 & $R$ & 68.3 & 65 & 49 & 2 & 2 & 2 & 2 & 2 & $L$ & 75.0 & 93 & 30 & 1 & 2 & 1 & 2 & 2 \\
\hline 7 & $R$ & 49.5 & 57 & 30 & 1 & 1 & 2 & 2 & 1 & $\mathrm{~L}$ & 78.0 & 99 & 28 & 5 & 3 & 2 & 2 & 2 \\
\hline 8 & $\mathrm{~L}$ & 58.5 & 83 & 60 & 4 & 3 & 2 & 2 & 1 & $\mathrm{R}$ & 77.0 & 87 & 33 & 1 & 2 & 1 & 1 & 2 \\
\hline
\end{tabular}

Table 3. Summary of results of quantitative variables.

\begin{tabular}{c|c|c|c}
\hline Variable & Autograft $(\mathbf{n}=\mathbf{8})$ & Bioactive glass $(\mathbf{n}=\mathbf{8})$ & $\mathbf{p}$ \\
\hline Dimension $\left(\mathbf{m m}^{2}\right)$ & & & 0.142 \\
\hline Mean (SD) & $63.5(8,4)$ & $70.5(7.0)$ & \\
\hline Median (min; max) & $64(49.5 ; 77)$ & $71.6(60 ; 78)$ & \\
\hline Osteoblasts & & & \\
\hline Mean (SD) & $65.1(11.7)$ & $93(20.5)$ & 0.037 \\
\hline Median (min; max) & $65.5(49 ; 83)$ & $96(59 ; 120)$ & \\
\hline Osteocytes & & & 0.025 \\
\hline Mean (SD) & $49.4(16.2)$ & $30.5(13.4)$ & \\
\hline Median (min; max) & $54.5(21 ; 63)$ & $31.5(12 ; 55)$ & \\
\hline Osteoclasts & & & $0.233^{*}$ \\
\hline Mean (SD) & $2(1.3)$ & $1.1(1.6)$ & \\
\hline Median (min; max) & $1.5(1 ; 4)$ & $1(0 ; 5)$ & \\
\hline Result of the paired Student t- test * * result of the paired Wilcoxon test.
\end{tabular}

found between the two groups is in accordance with the results of previous studies. . $^{10,17,18}$ Neovascularization plays a key role in bone repair; when it is modest or absent, bone neoformation in hindered. It has been already shown that bioactive glass can promote neovascularization, ${ }^{10,19}$ however, comparison to autograft had never been made before. The present study demonstrated that the neovascularization intensity between the two groups Acta Ortop Bras. 2015;23(4):202-7

\begin{tabular}{|c|c|c|c|}
\hline Variable & Autograft $(n=8)$ & Bioactive glass $(n=8)$ & $p$ \\
\hline Bone neoformation $\mathrm{n}(\%)$ & & & 0.480 \\
\hline 0 & $0(0.0)$ & $0(0.0)$ & \\
\hline 1 & $2(25.0)$ & $0(0.0)$ & \\
\hline 2 & $3(37.5)$ & $5(62.5)$ & \\
\hline 3 & $3(37.5)$ & $3(37.5)$ & \\
\hline Inflammatory infiltrate $\mathbf{n}(\%)$ & & & 0.059 \\
\hline 0 & $0(0.0)$ & $1(12.5)$ & \\
\hline 1 & $3(37.5)$ & $6(75.0)$ & \\
\hline 2 & $5(62.5)$ & $1(12.5)$ & \\
\hline 3 & $0(0.0)$ & $0(0.0)$ & \\
\hline Neovascularization $\mathrm{n}(\%)$ & & & $>0.0999$ \\
\hline 0 & $1(12.5)$ & $0(0.0)$ & \\
\hline 1 & $1(12.5)$ & $3(37.5)$ & \\
\hline 2 & $6(75.0)$ & $5(63.5)$ & \\
\hline 3 & $0(0.0)$ & $0(0.0)$ & \\
\hline Fibrosis n (\%) & & & 0.655 \\
\hline 0 & $0(0.0)$ & $0(0.0)$ & \\
\hline 1 & $3(37.5)$ & $2(25.0)$ & \\
\hline 2 & $5(63.5)$ & $6(75.0)$ & \\
\hline 3 & $0(0.0)$ & $0(0.0)$ & \\
\hline
\end{tabular}

Result of the paired Student t- test; * ${ }^{*}$ esult of the paired Wilcoxon test. 


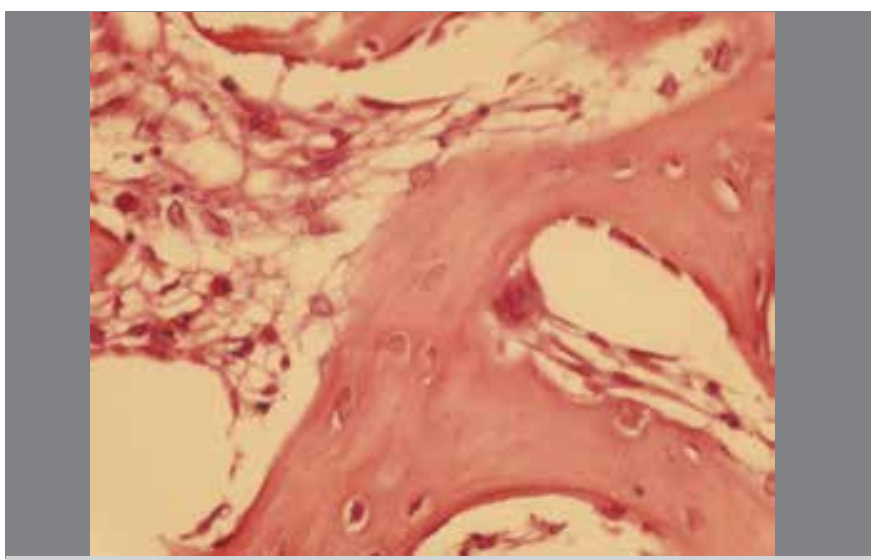

Figure 6. Example of newly formed trabecula in a defect treated with autograft, where many osteoblasts can be seen on the surface. It is also shown an osteoclast, in the center. HE staining, 400x magnification (rabbit 4, right side).

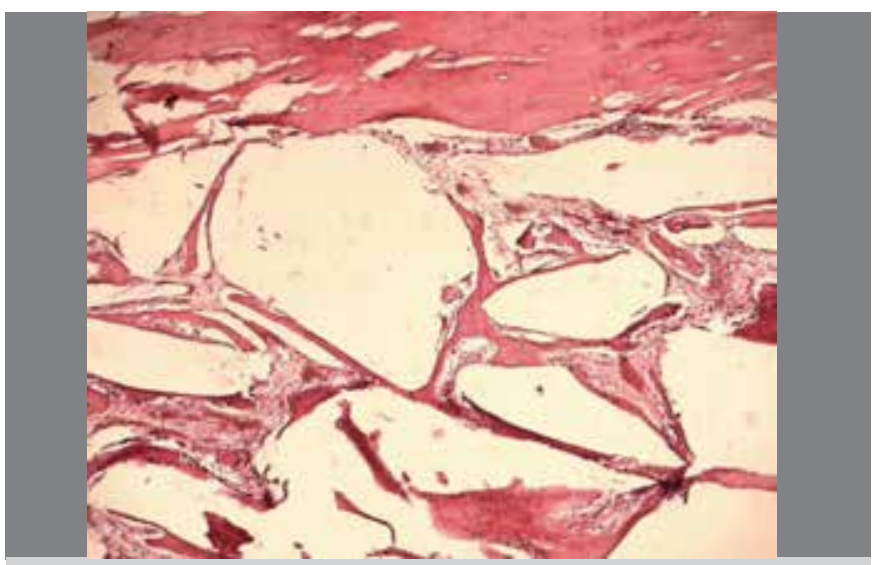

Figure 7. Femur treated with bioactive glass. At the top of the image cortical bone is shown, and below the remaining beads. Such beads are fully covered by newly formed trabeculae. HE staining, 50x magnification (rabbit 6, left side).

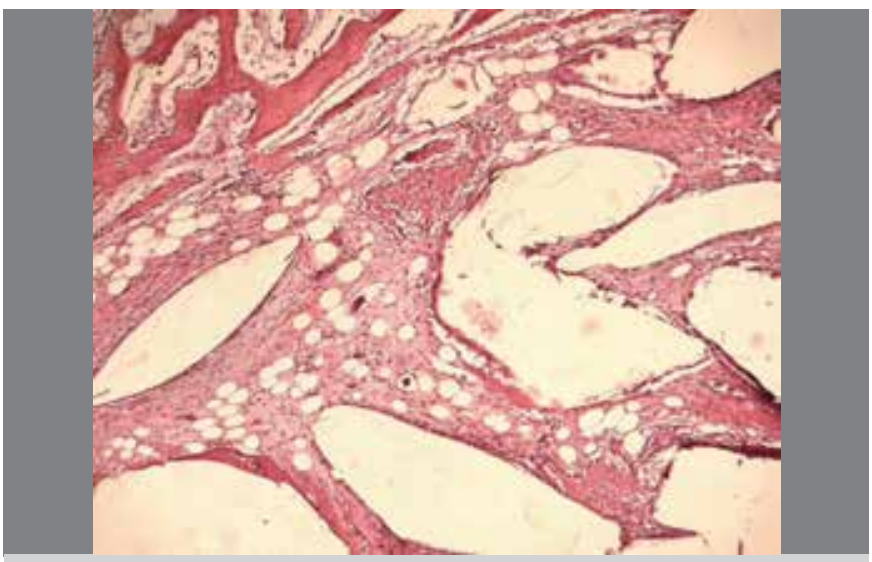

Figure 8. Femur treated with bioactive glass. In the upper left corner there is a bone neoformation area; in the whole image the remnants of the beads are shown. They are covered by a thin layer of newly formed trabeculae and among them, fibrous tissue and neoformed blood vessels. HE staining, 100x magnification (rabbit 7, left side).

was similar. The intensity of the inflammatory infiltrate was also similar between the two groups (even with a small trend to be lower in the bioactive glass group), showing that the granules do

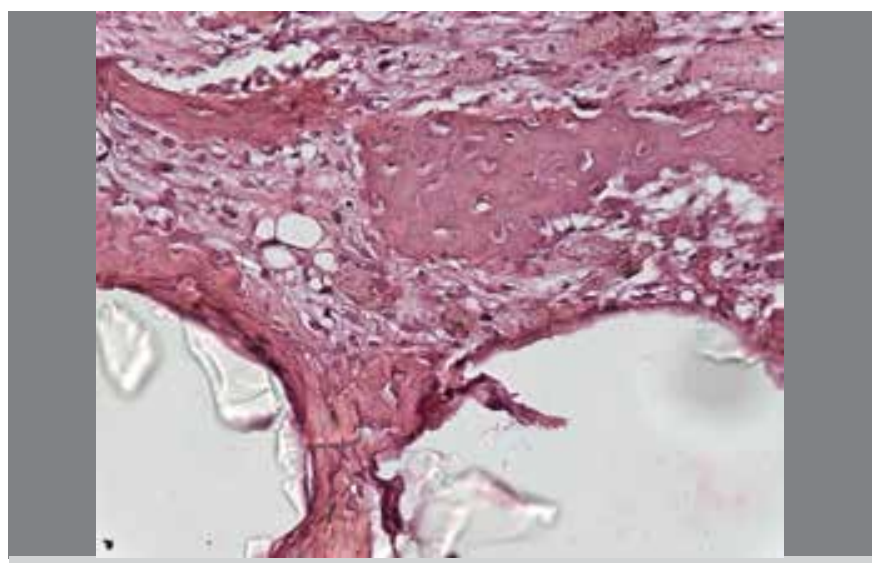

Figure 9. Femur treated with bioactive glass, with intense bone neoformation around the beads and newly formed blood vessels. Below we can see remaining fragments of beads. HE staining, increased 200x magnification (rabbit 8 , right side).

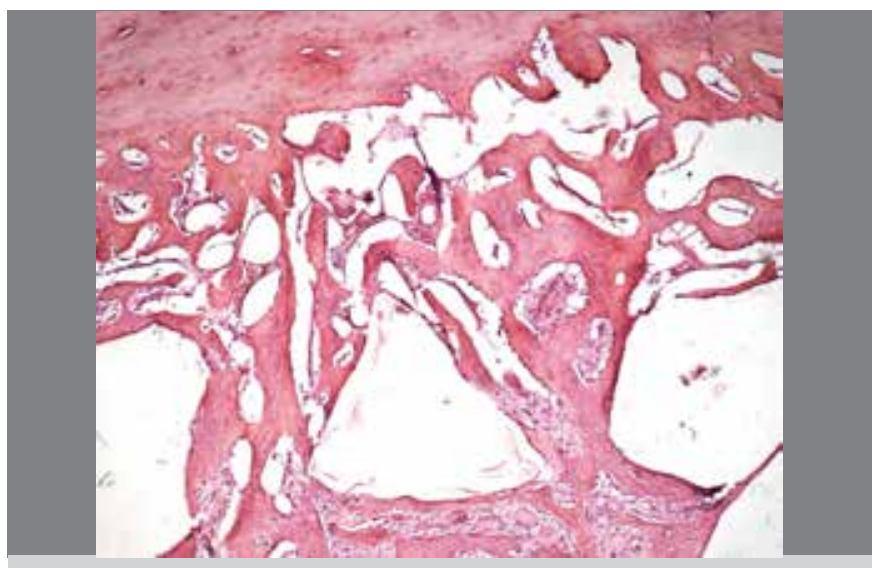

Figure 10. Femur treated with bioactive glass. Area with intense bone formation, next to one of the edges of the injury (cortical bone shown above). The presence of remnants of bioactive glass granules, fully covered by a layer of neoformed immature bone. HE staining, 100x magnification (rabbit 8, right side).

not induce an exacerbated inflammatory response, even being a foreign body, showing, thus, its biocompatibility. Finally, the intensity of fibrosis was also assessed in this study. Fibrosis appears in the first stages of bone consolidation (fibrous callus), thus, a different intensity between the two groups would mean that they were in different stages of the consolidation process, or that the repair speed or bone consolidation are different in both groups. However, fibrosis intensity was similar in both groups. These results indicate that the bioactive glass is very promising as a synthetic bone substitute.

This was the first study that carried out the count of the three fundamental cellular types of the bone metabolism: osteoblasts, osteocytes, and osteoclasts. Bioactive glass induces local bone increase, ${ }^{7}$ as previously explained. Therefore, it would be expected that the number of osteoblasts and osteoclasts were greater in the bioactive glass group than in the autograft group. The granules of bioactive glass, besides promoting increased osteoblast function, stimulate the growth and maturation of osteoblasts and promote the expression and maintenance of the osteoblast phenotype, delaying the differentiation into osteocite. ${ }^{7}$ Therefore, it 
would be expected that osteocyte count was not increased when compared to the autograft group, or would even be reduced. The results obtained in this study are in compliance with such expectations. The number of osteoblasts was nearly $50 \%$ higher in the bioactive glass group, and the number of osteocytes was nearly $30 \%$ of the amount found in the autograft group.

The finding regarding differences between numbers of osteoblasts and osteocytes corroborates the theory that bioactive glass increases bone turnover, stimulating osteoblasts and delaying its differentiation in osteocytes.

Regarding osteoclasts, we expected an increase in the group treated with bioactive glass granules for the same reasons (increase of bone turnover). However, we did not find any difference in the number of osteoclasts. This is probably due to the fact that the amount of osteoclasts is considerably lower than osteocytes or osteoblasts, thus, it would be necessary a much higher number of rabbits in order to find any difference between the two groups. Another alternative would be to alter the count area, since in the area used in this study (area of $0.17 \mathrm{~mm}^{2}$ with the greater increase objective) there were only one or two osteoblasts, or even none. However, if the count would have been made considering the total area of the injury, the number would be more significant in order to allow comparison (but practically it would invalidate manual count and automated count with proper software would become necessary).
Although some clinical trials have already been carried out (mainly case series) $)^{10,18}$ there is still space for molecular biology studies in order to clarify more details of the mechanism of action of bioactive glass. How do small alterations in granules constitution, as the presence of other ions, can alter this process? How do joint antiresorptive therapy, which would inhibit osteoclasts (for example, zoledronic acid), would affect bone neoformation? Is it possible to overlay implants with bioactive glass, just like some implants recovered by HA? What would be the difference in implants osseointegration (metal prostheses, for example) covered with bioactive glass or HA?

These and other questions show that this is still a gray area of medical knowledge, and much can be produced both in the experimental scope, as well as in pre-clinical and clinical studies.

\section{CONCLUSIONS}

Bioactive glass, when used to fill out cavitary defects in rabbits, presented a higher number of osteoblasts and a lower number of osteocytes when compared to autograft. Regarding the other variables, the two methods were similar.

\section{ACKNOWLEDGEMENTS}

The authors are deeply indebted to Ossis Medical Comércio Importação e Exportação Ltda, São Paulo, SP, Brazil, for the donation of bioactive glass granules.

\section{REFERENCES}

1. Zabeu JLA, Mercadante MT. Substitutos ósseos comparados ao enxerto ósseo autólogo em cirurgia ortopédica - revisão sistemática da literatura. Rev Bras Ortop. 2008;27;43(3):59-68.

2. Phillips GO. Clinical applications of bone allografts and substitutes. World Scientific Pub Co Inc; 2005.

3. Montgomery DM, Aronson DD, Lee CL, LaMont RL. Posterior spinal fusion:allograft versus autograft bone. J Spinal Disord. 1990;3(4):370-5.

4. Banwart JC, Asher MA, Hassanein RS. Iliac crest bone graft harvest donor site morbidity. A statistical evaluation. Spine (Phila Pa 1976). 1995;20(9):1055-60.

5. Ahlmann E, Patzakis M, Roidis N, Shepherd L, Holtom P. Comparison of anterior and posterior iliac crest bone grafts in terms of harvest-site morbidity and functional outcomes. J Bone Joint Surg Am. 2002;84(5):716-20.

6. Heikkilä JT, Mattila KT, Andersson O, Knuuti J, Yli-Urpo A. Behavior of bioactive glass in human bone. Bioceramics. 1995;8:35-41.

7. Välimäki VV, Aro HT. Molecular basis for action of bioactive glasses as bone graft substitute. Scand J Surg. 2006;95(2):95-102.

8. Hamadouche M, Sedel L. Ceramics in orthopaedics. J Bone Joint Surg Br. 2000;82(8):1095-9.

9. Virolainen P, Heikkilä J, Yli-Urpo A, Vuorio E, Aro HT. Histomorphometric and molecular biologic comparison of bioactive glass granules and autogenous bone grafts in augmentation of bone defect healing. J Biomed Mater Res. 1997;35(1):9-17.

10. Frantzén J, Rantakokko J, Aro HT, Heinänen J, Kajander S, Gullichsen E,et al Instrumented spondylodesis in degenerative spondylolisthesis with bioactive glass and autologous bone: a prospective 11-year follow-up. J Spinal Disord Tech. 2011;24(7):455-61.

11. Loty C, Sautier JM, Tan MT, Oboeuf M, Jallot E, Boulekbache H, et al. Bioactive

Acta Ortop Bras. 2015;23(4):202-7 glass stimulates in vitro osteoblast differentiation and creates a favorable template for bone tissue formation. J Bone Miner Res. 2001;16(2):231-9.

12. Munukka E, Leppäranta $O$, Korkeamäki $M$, Vaahtio $M$, Peltola $T$, Zhang $D$, et al. Bactericidal effects of bioactive glasses on clinically important aerobic bacteria. J Mater Sci Mater Med. 2008;19(1):27-32.

13. Leppäranta O, Vaahtio M, Peltola T, Zhang D, Hupa L, Hupa M, et al. Antibacterial effect of bioactive glasses on clinically important anaerobic bacteria in vitro. J Mater Sci Mater Med. 2008;19(2):547-51.

14. Lindfors NC, Aho AJ. Granule size and composition of bioactive glasses affect osteoconduction in rabbit. J Mater Sci Mater Med. 2003;14(4):365-72.

15. Rosselli JEGC. Estudo do efeito da sinvastatina na reparação óssea de cavidades cirúrgicas em fêmur de coelhos [tese]. São Paulo: Universidade Federal de São Paulo; 2012.

16. Kirkwood B, Sterne J. Essential Medical Statistics. 2nd ed. Massachussets (USA): Blackwell Science; 2006.

17. Heikkilä JT, Aho HJ, Yli-Urpo A, Happonen RP, Aho AJ. Bone formation in rabbit cancellous bone defects filled with bioactive glass granules. Acta Orthop Scand. 1995;66(5):463-7

18. Lindfors NC, Koski I, Heikkilä JT, Mattila K, Aho AJ. A prospective randomized 14-year follow-up study of bioactive glass and autogenous bone as bone graft substitutes in benign bone tumors. J Biomed Mater Res B Appl Biomater. 2010;94(1):157-64

19. Peltola MJ, Aitasalo KM, Suonpää JT, Yli-Urpo A, Laippala PJ. In vivo model for frontal sinus and calvarial bone defect obliteration with bioactive glass S53P4 and hydroxyapatite. J Biomed Mater Res. 2001;58(3):261-9. 